\title{
Risk Identification based on Hidden Semi-Markov Model in Smart Distribution Network
}

\author{
Fangyuan Chang, Wanxing Sheng, Tianshu Zhang, Yu Zhang, Xiaohui Song \\ Power Distribution Department, China Electric Power Research Institute, Beijing, China \\ Email: zhangtianshu@epri.sgcc.com.cn
}

Received April, 2013

\begin{abstract}
The smart distribution system is the critical part of the smart grid, which also plays an important role in the safe and reliable operation of the power grid. The self-healing function of smart distribution network will effectively improve the security, reliability and efficiency, reduce the system losses, and promote the development of sustainable energy of the power grid. The risk identification process is the most fundamental and crucial part of risk analysis in the smart distribution network. The risk control strategies will carry out on fully recognizing and understanding of the risk events and the causes. On condition that the risk incidents and their reason are identified, the corresponding qualitative / quantitative risk assessment will be performed based on the influences and ultimately to develop effective control measures. This paper presents the concept and methodology on the risk identification by means of Hidden Semi-Markov Model (HSMM) based on the research of the relationship between the operating characteristics/indexes and the risk state, which provides the theoretical and practical support for the risk assessment and risk control technology.
\end{abstract}

Keywords: Risk Identification; Hidden Semi-Markov Models; Smart Distribution Network

\section{Introduction}

With the continued growth of the economy, the social demand for electricity is at a critical turning point. In the future, both the scale and the complexity of structure are increasing for the smart distribution network. Along with the tremendous benefit in the development of the distribution network, the greater potential risk is also need to bear.

The distribution network locates at the end of the power system, which directly connects to the end-users. Therefore, the grid reliability and the user reliability are directly related. According to incomplete statistics, $80 \%$ to $95 \%$ of the user's outage is caused by the failure of the distribution network [1]. With the rapid development of the national economy and the improving demand for industrial and residential load, the challenges of reliability increases as well. Distribution network failure, which causes the power outages, will interrupt the users and brings economic losses. In order to analyze and prevent the accidents, the risk identification, risk assessment, and the appropriate risk control measures dominates very significant meaning nowadays.

In recent years, the study of distribution network risk analysis focuses on the risk assessment, which based on the uncertainties from distribution network, qualitatively or quantitatively gives a comprehensive measure of the likelihood and severity, while the risk identification is usually overlooked and affects the validity and accuracy of the risk assessment. The risk identification process is to determine the risk factors in the particular system and define its characteristics. The risk identification is the foundation and critical part in the risk analysis process. The risk identification process includes two main aspects: Firstly, find the sources of risk; secondly, identify the conditions of risk factors transformed to the accident.

In this paper, a risk identification method based on HSMM for the smart distribution network is presented. Firstly we study the mechanism of smart distribution network and divides the risk types for finding the sources of risk, then we establish the stochastic process model of the risk state and the operating characteristics, which identifies the accident transformed conditions from the risk factors to the risk accidents, finally, the risk identification model based on HSMM for the smart distribution is proposed, which helps to predict and identify risk effectively.

\section{Literature Research}

\subsection{Risk Assessment}

The aim of operating risk assessment in distribution network is to evaluate the exposure level of disturbance, which includes both the likelihood and severity of dis- 
turbance events. The research on the risk alert field abroad mainly focuses on the safety assessment, reliability evaluation and risk assessment. In the risk assessment of power system, the current research results can be divided into system-level and component-level. The risk assessment on trolly wires [2] and transformers [3] are in the component-level, while the system-level case includes risk assessment on the transient stability analysis [4], voltage stability analysis [5] and security range [6]. Literature[7] proposed a series functions to represent the severity of power flow overloading, generatrix low voltage, voltage instability, cascading overload, which is applied in the risk assessment of transmission system, has not in the field of distribution network.

\subsection{Markov Process}

In probability theory and statistics, a Markov process, is a stochastic process satisfying a certain property [8].A hidden semi-Markov model (HSMM) is a statistical model with the same structure as a hidden Markov model except that the unobservable process is semi-Markov rather than Markov. This means that the probability of there being a change in the hidden state depends on the amount of time that has elapsed since entry into the current state. This is in contrast to hidden Markov models where there is a constant probability of changing state given survival in the state up to that time [9].

This feature of the Markov process is usually applied to the analysis of state probability and system reliability after a self-healing power system's restoration. However, the basic conditions for applying the Markov process to get the reliability index are: the lifecycle distribution of the components of the system and the repair time distribution after the failure occur, and the other relevant distribution is exponentially distributed, and all the random variables are independent of each other.

At present, lots of research has been done base on this assumption. Literature [10] proposed a component outage model based on the Markov process, which combines the duration of the frequency to perform the reliability analysis for small system. Literature [11] presented a transformer reliability assessment model based on Markov process. However, in practice, such as the com- ponent reliability assessment, if the lifecycle and repair time distribution are not exponential distribution, the process is far from the Markov process, and will reduce the accuracy of the simulation results. The Semi-Markov process does not require the exponential distribution assumption on the transfer function and widely used to the power plant reliability assessment [12], the uninterruptible power supply (USP) reliability assessment [13] and the protective relay reliability assessment [14]. In particular, literature [15] proposed equipment failure prediction method based on HSMM to predict the probability of each state according to the partial observation predilection system.

\section{Risk Identification based on HSMM}

If the time is ordered by $t_{1}<t_{2}<\ldots<t_{n}$ in data set $\left(t_{1}, t_{2}, \ldots, t_{n}\right)$, the distribution function of $X\left(t_{n}\right)=x_{n}$ is as follows:

$$
F_{X_{n} \mid X_{X} X_{2} \cdots X_{N-1}}=F_{X_{N} \mid X_{N-1}}
$$

while $X\left(t_{i}\right)=x_{i}, \quad i=1,2, \cdots, n-1$

This random process of this nature is defined a Markov process which follows the principles: the random process at the time of $t>t_{0}$ is already known, the process at $t_{0}$ is only relevant to the process at while irreverent to the process before $t_{0}$, which is also called nonmemory property or no-follow-up effect, in simple, the future development of given state process is independent of probability rules in the history.

This paper presented a Hidden Semi-Markov Model (HSMM) for the risk prediction in smart distribution system. The HSMM described two stochastic processes; one is the semi-Markov process with randomness to describe the transferring relationship between the states, while another is to describe the stochastic relationship of states and observation values.

Assuming the state data set is $\left(q_{1}, q_{2}, \cdots q_{L}\right)$, and the corresponding observation data set is

$$
\left[\left(o_{1}, \cdots o_{l_{1}}\right), \cdots\left(o_{l_{L-1}+1}, \cdots o_{L}\right)\right] \text {, }
$$

the $t^{\text {th }}$ state $q_{t}$ observation data set is $\left(o_{l_{L-1}+1}, \cdots o_{L}\right)$, and the state duration is $l_{t}-l_{t-1}$. The Figure 1 shows the process of the hidden semi-Markov model.

\section{Scheme of Risk Identification based on HSMM}

Aiming at the limitation of current research of risk in the distribution network, this paper propose the concept of risk identification, reveals the cause of risk which is the

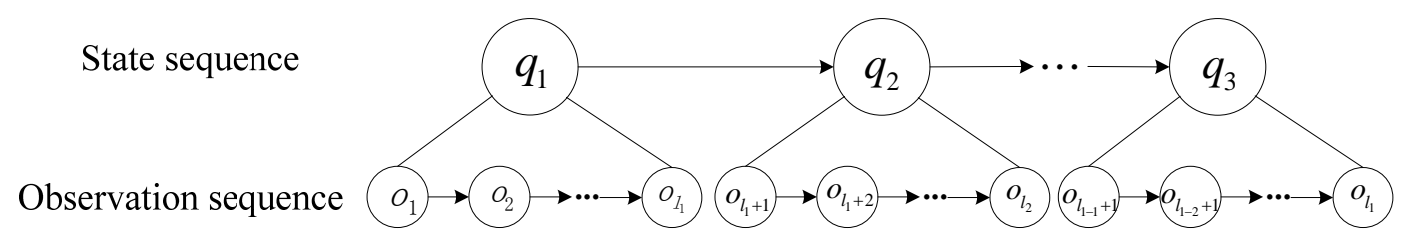

Figure 1. The process of the hidden semi-Markov model. 
relationship of the operating characteristics/indexes before and after the risk's occurrence, on the other hand, the state transferring principles of component / system is presented to provide reference basis for risk prevention and control. The specific research scheme is shown in Figure 2 and the steps are as follows:

- Step 1: study the risk diversity in smart distribution network according to the actual situation, analysis the possible risks in smart distribution network;

- Step 2: According to the type of risk, identify the state of component / system, establish the state space. The state space is defined as normal state, overload state, overvoltage state, low-voltage state and failure state shown in Figure 3:

- Step 3: Select a real distribution network, monitor the frequency of cases occurs within a certain period, and the abnormal information /operating characteristics /index before the incidents;

- Step 4: obtain the state transition probability matrix by means of neural network training method;

- Step 5: According to the results of Step 2 and Step 4, establish the state transition model based on the Markov process risk;

- Step 6: eliminate the abnormal information / operating characteristics /index irrelevant to the incidents using the theoretical analysis, mathematical statistics method, and then apply the multi-source information fusion to get the relevancy of risk and operating characteristics/indexes observed;

- Step 7: According to the results of step 6, establish the stochastic process model of risk state and values obrved;

- Step 8: combine the results of Step 5 and Step 7, establish the risk prediction model based on HSMM of smart distribution network;

- Step 9: verify the model above by the dynamic simulation test, and validate the model according to the test results.

The risk identification method introduced is different from the traditional research in the operation indexes, according to the steps above, with the data mining and multi-dimensional method, the large number of history/ real-time monitoring/simulation information in the smart distribution network are applied to analyze the relationship between the risks and operation characteristics of the distribution network, propose the risk identification model base on HSMM and predict the possible risks that the system may face according to the current component/ system states.

\section{Future Works and Conclusions}

In this paper, on the base of the establishment of the risk state transition probability model and the random process model of the risk status and monitoring values, the mechanism of distribution network risk is revealed theoretically, the smart distribution network risk identification model is proposed as well, providing the new ideas for risk warning from the nature and mechanism of risk. The research achievement can be



Figure 2. The specific research scheme on the risk identification based on HSMM. 


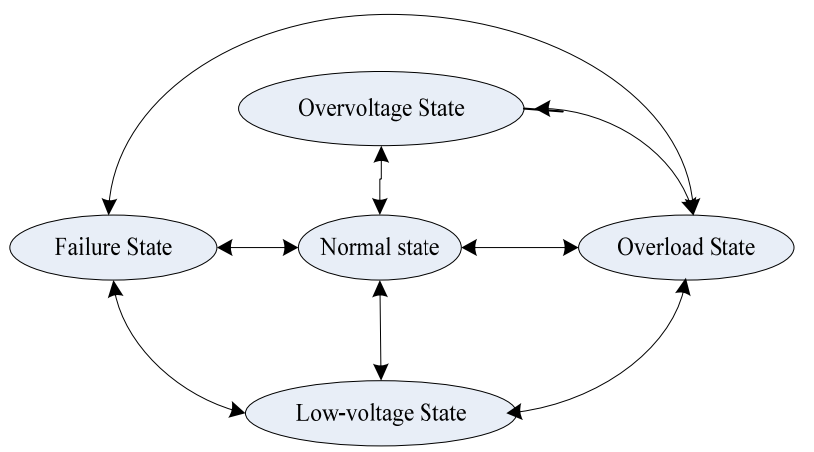

Figure 3. The proposed state transfer model.

directly used to the theoretical basis of smart distribution network risk warning. For the practical application, the risk prediction analysis and causes mining before the incident provides technical support for smart distribution network from passive defense to active defense, which promotes the security of electricity supply, improve the reliability, and reduces the impact of the grid accident and hazards, and has great practical significance on operation, planning and designing of the power system and the development of society as well.

\section{Acknowledgements}

This work is supported by Active defense Technology based on Multi-source Information Fusion of Smart Distribution Network of State Gird Cooperation of China (SGCC) and Risk Alert Technology based on Multisource Information Fusion in Smart Distribution Network (Project No. 51177152) of National Science Foundation of China.

\section{REFERENCES}

[1] R. Billinton and P. Wang, "Reliability Network Equivalent Approach to Distribution System Reliability Evaluation," IEEE Proceedings: Generation, Transmission and Distribution, Vol. 145, No. 2, 1998, pp. 149-153.

[2] R. X. Liu, J. H. Zhang and D. Wu, "Research on Static Security Index of Distribution Network Based on Risk Theory," Power system Protection and Control, Vol.39, No.15, 2011, pp. 89-95.

[3] H. Wan, J. D. Mccalley and V. Vittal, "Increasing Thermal Rating by Risk Analysis," IEEE Trans. on Power Systems, Vol. 14, No. 3, 1999, pp. 815-828. doi:10.1109/59.780891

[4] W. H. Fu, J. D. Mccalley and V. Vittal, "Risk Assessment for Transformer Loading," IEEE Transactions on Power Systems, Vol. 16, No. 3, 2001, pp. 346-353. doi:10.1109/59.932267

[5] M. Ni, J. D. Mccalley and V. Vittal, "Software Implementation of Online Risk-Based Security Assessment," IEEE Trans on Power Systems, Vol. 18, No. 3, 2003, pp. 1165-11728. doi:10.1109/TPWRS.2003.814909

[6] H. Wan, J. D. Mccalley and V. Vittal, "Risk based Voltage Security Assessment," IEEE Transactions on Power Systems, Vol. 15, No. 4, 2000, pp. 1247-1254. doi:10.1109/59.898097

[7] J. D. Mccalley, A. A. Fouad and V. Vittal, “A Risk-Based Security Index for Determining Operating Limits in Stability-Limited Electric Power Systems,” IEEE Transactions on Power Systems, Vol. 12, No. 3, 1997, pp. 1210-1219.doi:10.1109/59.630463

[8] Wikipedia, “Markov Process,” 2013. http://en.wikipedia.org/wiki/Markov_process

[9] S. Z. Yu, "Hidden Semi-Markov Models," Artificial Intelligence, Vol. 174, No. 2, 2009, pp. 215-243. doi:10.1016/j.artint.2009.11.011

[10] W. Y. Li, "Risk Assessment of Power Systems: Models, Methods, and Applications,” 1st Edition, John Wiley \& Sons Ltd., Chichester, 2004. doi:10.1002/0471707724

[11] R. Jin, Z. N. Xiao and J. Gong, "Markov Model for Reliability Assessment of Power Transformers," High Voltage Engineering, Vol. 36, No. 2, 2010, pp. 322-328.

[12] M. Perman, A. Senegacnik and M. Tuma, "Semi-Markov models with an application to power-plant reliability analysis," IEEE Transactions on Power Reliability, Vol. 46, No. 4, 1997, pp. 526-532. doi:10.1109/24.693787

[13] Y. Liang, R. M. Fricks and K. S. Trivedi, “Application of semi-Markov process and CTMC to evaluation of UPS system availability,” Proceedings of 2002 Annual Reliability and Maintainability Symposium, Seattle, 28-31 January 2002, pp. 584-591. doi:10.1109/RAMS.2002.981706

[14] L. J. Wang, G. Wang and B. Li, "Reliability Modeling of a Protection System Based on the Semi-Markov Process," Automation of Electric Power Systems, Vol. 34, No. 18, 2010, pp. 6-10.

[15] X. Jun and X. Y. Xiao, “A Method of Reliability Cost Assessment in Distribution System Based on Semi-Markov Process,” Relay, Vol. 34, No. 12, 2006, pp. 57-62. 